\title{
STRUCTURE MALACOLOGIQUE DE LA RIVIERE AUBE
}

\author{
par J. MOUTHON 1
}

\begin{abstract}
Au cours d'un inventaire faunistique de la rivière Aube, 40 espèces de Mollusques ont été recensées (28 Gastéropodes et 12 Lamellibranches).

L'application de l'analyse factorielle des correspondances aux données ainsi obtenues a permis de vérifier l'existence d'une organisation des espèces (structure biologique) le long du cours d'eau puis d'établir une hiérarchisation des espèces le long de l'écosystème. La vicariance, le preferendum typologique, le degré d'euryécie des espèces peuvent ainsi être appréciés.
\end{abstract}

\section{Malacological structure of the river Aube}

Forty species of Mollusca are recorded from the river Aube (8 gasteropods and 12 bivalves.

A factorial analysis of correlations enabled us to describe the biological structure of the river and a hierarchy of species.

Vicariance, typological preferendum and ecological amplitude of species are thus estimated.

\section{1. - INTRODUCTION}

La place réservée aux Mollusques dans les travaux d'écologie potamique s'avère extrêmement réduite. Les inventaires malacologiques approfondis de systèmes d'eau courante sont très restreints, les relevés partiels se limitant généralement à des rivières de petites dimensions, parfois à caractère torrentiel, peu propices au développement de la faune malacologique (Thienemann 1911, 1912, 1926, Tomaszewski 1928, Geisjkes 1936, Nietzke 1937, Kuhn 1940, Fittkau 1949, Albrecht 1952, Illies 1952, Dittmar 1955, Hinz 1968, Fellenberg 1968, Armitage Mac Hale et Crisp 1975, Thiel et Subklew 1976) ; ces travaux ne concernent que rarement des systèmes d'eau courante plus importants comprenant Rhithron et Potamon (Beyer 1932, Shadin 1935, Hässlein 1956, Bonazzi et Ghetti 1977).

1. Laboratoire d'Hydroécologie du C.T.G.R.E.F. (Paris) et Centre d'Hydrobiologie de l'Université de Franche-Comté, place du Maréchal-Leclerc, 25030 Besançon Cedex.

Je remercie M. J.G.J. Kuiper pour l'aide précieuse qu'il ma apportée dans l'approche de la connaissance systématique des Pisidies, pour avoir bien voulu vérifier mes déterminations et relire mon manuscrit. 
Plus nombreux sont les documents présentant des groupements d'espèces associés à des biotopes particuliers commes les sources (Bornhauser 1912, Schermer 1922, Schuster 1925, Thienemann 1926, Beyer 1932, Kuhn 1940, Nadig 1942, Feldmann 1975) ou des secteurs de rivières de modeste importance (Geyer 1911, Miegel 1961 et 1964, Feldmann 1972, Kinzelbach 1972, Kinzelbach et coll. 1972, Botosaneanu et Negrea 1976, Blœsch 1977).

La majorité des travaux actuels concernant les eaux courantes sont consacrés à l'étude de groupes d'espèces appartenant à une même famille : Ancylidae (Maitland 1965, Calow 1973 et 1974, Durrant 1977), Lymnaeidae (Mc Craw 1970, Young 1975), Physidae (Frömming 1950, Young 1975), Planorbidae (Calow 1974 et 1975, Young 1975), Hydro. biidae (Cherbonnier 1952, Schäfer 1953, Lucas 1960, Bernasconi 1967 à 1975, Bœters 1973, Radoman 1976, Jungbluth 1972), Valvatidae (Young 1975), Margaritanidae (Hendelberg 1959, Jungbluth 1976), Unionidae (Mentzen 1926, Negus 1966, Norelius 1967, Wolff 1968, Haukioja et Hakala 1974, Modell 1974), Sphaeriidae (Kuiper 1949, Dance 1957 et 1970, Ladle-Baron 1969, Kuiper et Wolff 1970, Feldmann 1971 à 1977, Meier-Brook 1975, Bishop et Hewitt 1976).

La synthèse de telles données autécologiques, fragmentaires et parfois contradictoires, s'avère délicate et ne permet pas actuellement d'établir la répartition écologique des mollusques dans les eaux courantes ni de définir des groupements écologiques d'espèces. Lors de l'établissement d'une diagnose écologique de l'Aube préalable à la mise en service du "Réservoir Aube», nous avons été conduit à inventorier les mollusques de cette rivière, puis à traiter les données ainsi obtenues par une méthode statistique, l'analyse factorielle des correspondances, dans le but de vérifier l'existence d'une structure biolo. gique et éventuellement de définir les groupements d'espèces s'individualisant le long de l'écosystème considéré.

\section{2. - LE MILIEU}

\section{- Données géologioues et hydrologioues ( $f i g .1$ )}

L'Aube prend sa source sur la commune d'Auberive (Haute-Marne) dans le Jurassique moyen à une altitude de $379 \mathrm{~m}$. Après un parcours de $238 \mathrm{~km}$ orienté sensiblement S.E.-N.O., elle se jette dans la Seine à une altitude de $68 \mathrm{~m}$ en ayant traversé successivement les calcaires du Jurassique Supérieur, les terrains crayeux du Crétacé et dans son cours médian les marnes et argiles de Gault de la Champagne humide ; terrains imperméables qui ont permis la réalisation des grands barrages réservoirs "Seine " et "Marne ». 
La superficie de son bassin versant, marno-calcaire et crayeux, à vocation essentiellement agricole, est de $4670 \mathrm{~km}^{2}$.

Le régime hydrologique de l'Aube est caractérisé par des crues en fin d'automne et en hiver (décembre, janvier et février) ainsi que par des hautes eaux de moindre amplitude au printemps. A Blaincourt (bassin versant $1640 \mathrm{~km}^{2}$ ), le débit moyen mensuel pour la période 1956-1973 est de $17 \mathrm{~m}^{3} / \mathrm{s}$, à Arcis-sur-Aube (bassin versant $3590 \mathrm{~km}^{2}$ ) pour la période de référence $1960-1973$ il est de $29 \mathrm{~m}^{3} / \mathrm{s}$.

La période de basses eaux se situe de juillet à octobre, les débits journaliers pouvant devenir inférieurs à $1 \mathrm{~m}^{3} / \mathrm{s}$.

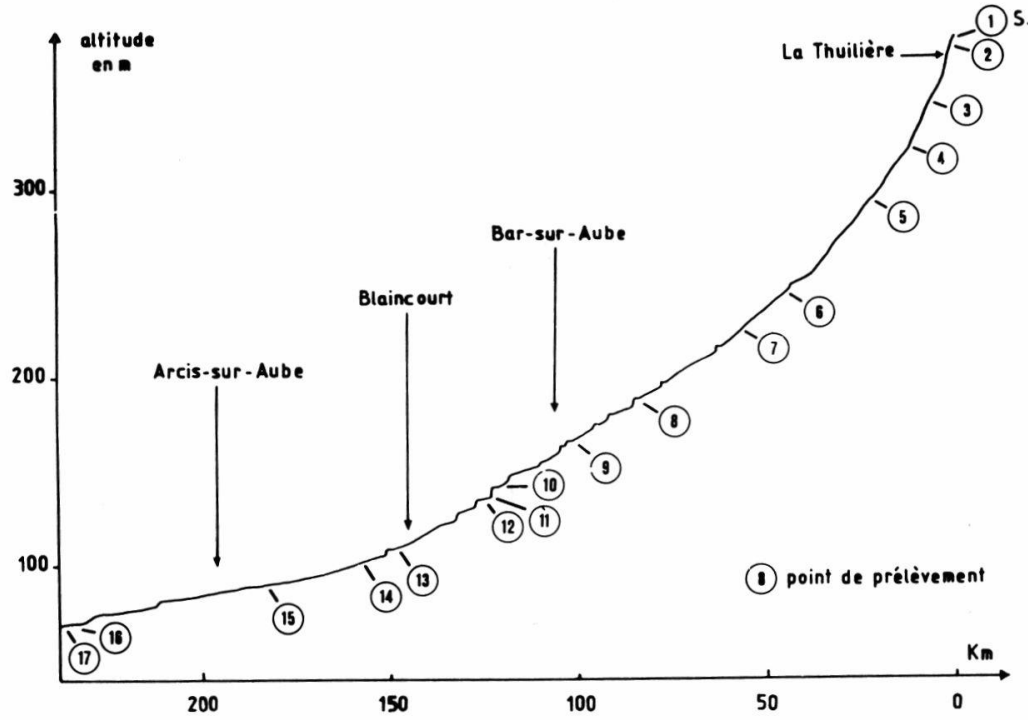

Fig. 1. - Profil longitudinal de l'Aube. Situation des points de prélèvement.

\section{- Données PHYSICO-CHIMIQUeS ${ }^{1}$}

Les principales caractéristiques physico-chimiques de ces eaux froides (fraîcheur due à l'alimentation par la nappe) calciques, alcalines, fortement minéralisées, riches en oxygène dissous mais aussi en nitrates et phosphates (associés à une activité agricole intense) sont mentionnées dans le tableau I.

1. Données S.R.A.E. Lorraine, S.R.A.E. Besançon, C.T.G.R.E.F. Paris, Lab. C.S.P. 


\begin{tabular}{|c|c|c|c|c|c|c|}
\hline $\mathrm{pH}$ & & $: \quad 7,15$ & 8,8 & O. dissous & $: \quad 6,4-12,2$ & \\
\hline $\mathrm{C}\left({ }_{\mu} \mathrm{S}\right)$ & & $: 285$ & 536 & $\%$ saturation & $: 57-124$ & \\
\hline $\mathrm{HCO}_{:}$ & $(\mathrm{mg} / \mathrm{l})$ & $: 216$ & -320 & Oxyd. & $: 0,2-8,5$ & \\
\hline $\mathrm{Ca} \mathrm{Mg}$ & $(\mathrm{mg} / \mathrm{l})$ & $: 64$ & -146 & $(\mathrm{mg} / \mathrm{l})$ & $0,5-\quad 4,5$ & \\
\hline $\mathrm{Cl}^{-}$ & $(\mathrm{mg} / \mathrm{l})$ & 7 & -16 & $\theta\left({ }^{\circ} \mathrm{C}\right)$ & $: 6,4-21$ & (source $8-9,5$ ) \\
\hline $\mathrm{SO}_{+}^{-}$ & $(\mathrm{mg} / \mathrm{l})$ & $: 10$ & $-14,6$ & $(\mathrm{mg} / \mathrm{l})$ & $4-9$ & \\
\hline $\mathrm{PO}_{4}^{-\cdots-}$ & $(\mathrm{mg} / \mathrm{l})$ & 0,3 & - 4,3 & $(\mathrm{mg} / \mathrm{l})$ & $: 2,4-5,9$ & \\
\hline $\mathrm{NO}_{3}^{-}$ & $(\mathrm{mg} / \mathrm{l})$ & 0,01 & -33 & $(\mathrm{mg} / \mathrm{l})$ & $: 1,1-1,5$ & \\
\hline $\mathrm{NO}_{-}^{-}$ & $(\mathrm{mg} / \mathrm{l})$ & $:$ & - $\quad 0,6$ & $(\mathrm{mg} / \mathrm{l})$ & $3,9-$ & \\
\hline $\mathrm{NH}_{4}^{+}$ & $(\mathrm{mg} / \mathrm{l})$ & 0 & - $1,4 i$ & & & \\
\hline
\end{tabular}

TABLEAU I. - Valeurs extrêmes des paramètres physico-chimiques.

Seules y figurent les valeurs extrêmes relevées sur l'ensemble du cours d'eau sans tenir compte de l'ordre des stations.

\section{- DonnÉEs TYPologiques (Tableau II)}

La recherche, grâce à l'analyse factorielle des correspondances, d'un nombre restreint de composantes typologiquement significatives

\begin{tabular}{|c|c|c|c|}
\hline Stations & $\begin{array}{l}\text { Niveau typologique } \\
\text { théorique calculé } \\
\text { (Verneaux 1977) }\end{array}$ & $\begin{array}{c}\text { Correspondance } \\
\text { avec } \\
\text { la classification } \\
\text { de Huet (1946-49) }\end{array}$ & $\begin{array}{c}\text { Correspondance } \\
\text { avec } \\
\text { la classification } \\
\text { de Illies (1953-63) }\end{array}$ \\
\hline 1 source & $<1$ & domaine non & MéSOCRENON 1 \\
\hline 2 aval source & 1,1 & piscicole & \\
\hline 3 amont Auberive & 3,5 & & \\
\hline 4 ferme Cadet & 4,7 & groupement supé. & EPIRHITHRON \\
\hline 5 Arbot & 5,2 & rieur à truite & MÉSORHITHRON \\
\hline 6 Boudreville & 6,4 & groupement infé- & \\
\hline 7 Veuxhalles & 6,1 & rieur à truite & HYPORHITHRON \\
\hline 8 Villes/la Ferté & 6,4 & (groupement & \\
\hline 9 Baycl & 6,8 & à ombre) & \\
\hline 10 Beaulieu (retenue) & 7,2 & & \\
\hline 11 aval retenue & - & & \\
\hline 12 Unienville & 7 & & \\
\hline 13 (noue Magnicourt) & - & groupcment & EPIPOTAMON \\
\hline 14 Epagne & 7,1 & à barbeau & \\
\hline 15 Saint-Narbor & 7,3 & & \\
\hline 16 Saron & 7,3 & & \\
\hline 17 Marcilly & 7,3 & & \\
\hline
\end{tabular}

1. Compléments proposés (Verneaux, 1973).

Tablead II. - Données typologiques théoriques des stations de I'Aube. 
et l'étude des variations de ces facteurs synthétiques le long de la structure biotypologique représentative de l'écosystème «eau courante" non pollué ont permis la sélection de paramètres discriminants: température moyenne du mois le plus chaud, dureté totale, distance aux sources, section mouillée à l'étiage, pente, et par suite, l'établissement de trois équations qui permettent de déterminer le type écologique théorique, ou potentiel typologique approché, de chaque station (Verneaux, 1977).

Les valeurs ainsi obtenues (tableau $\mathrm{n}^{\circ} 2$ ), vérifiées par l'analyse des groupements ichtyologiques et d'invertébrés benthiques (rapport C.T.G.R.E.F. 1978), permettent d'intégrer les recherches effectuées. dans un cadre typologique théorique dont les correspondances avec les classifications proposées par Huet (1949) et Illies (1963) ont été établies.

\section{3. - METHODES D'ECHANTILLONNAGE}

L'échantillonnage qualitatif et surtout quantitatif a toujours posé et pose encore (Hairston 1958) de sérieux problèmes aux Malacologistes ct l'on peut se rallier à l'opinion de Puton qui souligne dès 1847 que "la recherche des Mollusques demande des soins extrêmes et des perquisitions, dans les eaux, souvent pénibles ". Ceci tient à la fois à la diversité et à la complexité des microhabitats occupés par les Mollusques, aux importantes fluctuations saisonnières des populations et aux écarts de tailles qui, au sein d'une même classe, peuvent être considérables (de $2 \mathrm{~mm}$ pour $P$. tenuilineatum à $200 \mathrm{~mm}$ pour $A$. cygnaea) en présentant tous les intermédiaires possibles.

Tous les types d'habitat recensés sur une station doivent faire l'objet d'un échantillonnage quantitatif, préférentiellement en été, saison qui correspond à l'effectif maximum des populations, les techniques de prélèvement étant adaptées aux divers habitats reconnus en chaque station :

- BLOCS, PIERRES, GALETS, BOIS MORTS, sont prospectés sur $1 \mathrm{~m}^{2}$, aussi bien en faciès lotique que lénitique, les individus adhérant forte. ment aux supports, comme Ancylus fluviatilis, Theodoxus fluviatilis, Radix peregra, étant prélevés au moyen d'un scalpel.

- graviers, sables, limons, vases, sont échantillonnés au moyen d'un troubleau emmanché, de section rectangulaire (longueur $25 \mathrm{~cm}$ ) muni d'un filet avec un vide de maille de $630 \mathrm{~mm}$; l'appareil est tiré sur une longueur d'un mètre.

Les Unionidae prélevés au moyen du même instrument, ou à la benne Ekman au-delà de $1,5 \mathrm{~m}$ d'eau, sont estimés au $\mathrm{m}^{2}$ dans l'habitat ou leur abondance est maximale. 
- VÉGÉTAUX (HydRophytes ET AMPHIPHYTES) : tous les macrophytes présents sur la station ou plus précisément les habitats qu'ils constituent, sont prospectés; lorsque la plante est fixée, la tige est coupée à la base, puis lavée dans un filet de Surber ou un tamis à maille fine $(630 \mu)$. Toutefois, les différentes associations que ces espèces peuvent former entre elles, la distance au bord, la hauteur d'eau, constituent autant de microhabitats différents, qu'il convient de prendre en compte séparément.

Utilisée au cours de nombreuses études (Phytosociologie, Ecologie planctonique, Biotypologie), l'analyse factorielle des correspondances, dont les principes sont exposés en détail dans les ouvrages de Cordier 1965, Lebart et Fénelon 1971, Benezecri 1973, est employée ici afin de tester les possibilités typologiques éventuelles des Mollusques d'eau douce préalablement à des travaux d'écologie concernant les espèces de cet embranchement. L'utilisation des données brutes, des abon. dances relatives, ou plus simplement du critère présence-absence s'étant révélée insuffisante, nous avons utilisé des classes relatives d'abondance ( 5 classes) établies par genre ou groupe d'espèces morphologiquement et biologiquement voisines, en fonction des données bibliographiques d'une part et des connaissances acquises sur d'autres rivières (bassin du Rhône) d'autre part.

\section{4. - ANALYSE DU PEUPLEMENT}

40 espèces de Mollusques (Gastéropodes et Lamellibranches) ont été recensées sur l'Aube (Tableau III) ; rencontrés en un seul exemplaire, Pisidium hibernicum et Sphaerium lacustre ont été exclus de l'analyse.

\subsection{Distribution des espèces le long du cours d'eau (fig. 2)}

Dans le cas de l'étude des invertébrés benthiques d'une rivière, l'analyse de diagrammes représentant la distribution quantitative longitudinale des espèces permet d'apprécier :

- semi-quantitativement le remplacement des espèces de l'amont vers l'aval ( $R$. peregra - $R$. auricularia - $P$. fontinalis - $P$. acuta) ;

- leur degré d'eurytopie relativement au système considéré ; parmi les espèces eurytopes pour l'Aube, présentes sur la majeure partie du cours d'eau et ne pouvant servir à "caractériser » un secteur particulier, figurent : $P$. subtruncatum, $P$. nitidum, $P$. henslowanum, G. albus, B. tentaculata... ;

- les affinités entre espèces et stations ; ainsi $V$. piscinalis (stations 56-15) associée à la quantité des dépôts organiques, est remplacée 
1. Bythiospeum diaphanum Michaut, 21. Bithynia tentaculata Linné, 1758. 1831.

2. Bythinella carinulata Drouet, 1867.

3. Pisidium personatum Malm, 1855.

4. Radix peregra Müller, 1774.

5. Ancylus fluviatilis Müller, 1774.

6. Bathyomphalus contortus Linné. 1758.

7. Pisidium amnicum Müller, 1774.

8. Pisidium milium Held, 1836.

9. Valvata piscinalis Müller, 1774.

10. Galba palustris Müller, 1774.

11. Planorbis carinatus Müller, 1774.

12. Physa fontinalis Linné, 1758.

13. Pisidium nitidum Jenyns, 1832.

14. Lymnea stagnalis Linné, 1758.

15. Anisus vortex Linné, 1758.

16. Gyraulus albus Müller, 1774.

17. Pisidium subtruncatum Malm, 1855.

18. Myxas gutinosa Müller, 1774.

19. Pisidium henslowanum Sheppard, 1823.

22. Sphaerium corneum Linné, 1758.

23. Bithynia leachii Sheppard, 1823.

24. Unio crassus Philipsson, 1788.

25. Acrolaxus lacustris Linné, 1758.

26. Anodonta anatina Linné, 1758.

27. Planorbis corneus Linné, 1758.

28. Theodoxus fluviatilis Linné, 1758.

29. Unio pictorum Linné, 1758.

30. Radix auricularia Linné, 1758.

31. Pisidium supinum Schmidt, 1851.

32. Physa acuta Draparnaud, 1805.

33. Segmentina complanata Linné, 1758.

34. Pisidium casertanum forme ponde. rosa Stelfox, 1918.

35. Pisidium moitessierianum Paladilhe, 1866.

36. Armiger crista Linné, 1758.

37. Potanopyrgus jenkinsi Smith, 1889.

38. Viviparus viviparus Linné, 1758.

39. Galba truncatula Müller, 1774.

40. Pisidium casertanum Poli, 1791.

Tableau III. - Liste des espèces de Mollusques de l'Aube prises en compte pour l'analyse.

par B. tentaculata plus inféodée aux substrats pierreux (stations 9 13).

Toutefois, de tels graphiques, strictement descriptifs, se prêtent mal à la confrontation de données multiples et d'autre part ne permettent pas d'accéder aux connaissances rapportées à un espace abstrait. Le traitement d'inventaires faunistiques portant sur un grand nombre de systèmes aquatiques (études en cours sur le bassin du Rhône) néces. site l'emploi de méthodes d'analyse mathématique synthétique, comme l'analyse factorielle des correspondances qui a été expérimentée ici.

\subsection{Organisation des espèces dans une analyse factorielle}

La distribution des espèces et des stations dans le plan des deux premiers axes $\left(F_{1}, F_{2}, 43,4 \%\right.$ d'inertie expliquée) s'organise en une succession, aux accidents structuraux près expliqués par ailleurs, des espèces et corrélativement des stations, selon une courbe en U inhérente à la méthode, analogue à celles obtenues antérieurement au 
B. diaphanum

B. carinulata

V. piscinalis

B. tentaculata

P. jenkinsi

T. fluviatilis

V. viviparus

A. fluviatilis

A. lacustris

R. peregra

$R$. auriculario

$P$. fontinalis

P. acuta

L. stagnalis

G. palustris

G. truncatula

B. contortus

G. albus

P. carinatus

A. vortex

P. corneus

U crassus

U. pictorum

A. anatina

$S$. corneum

P. personatum

P. casertanum

P. subtruncatum

P nitidum

P. amnicum

$P$. milium

P. henslowanum

P. supinum

$P$ moitessierianum

P. ponderosum
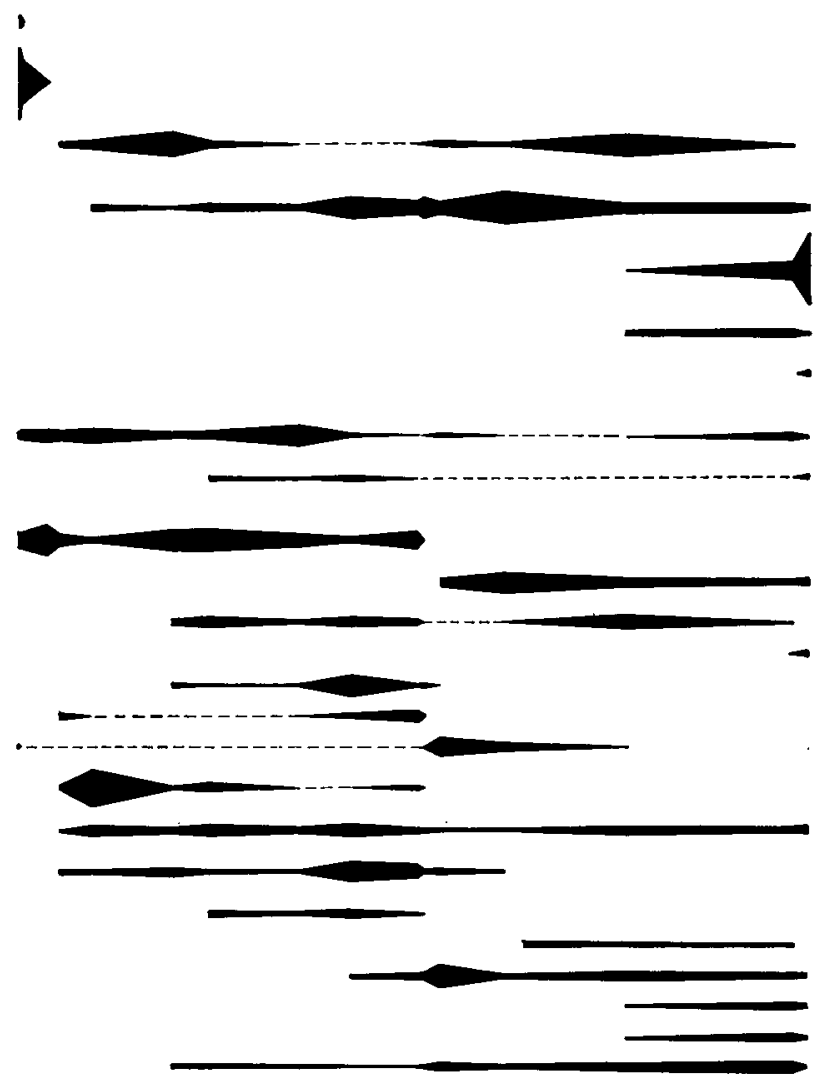

$-$

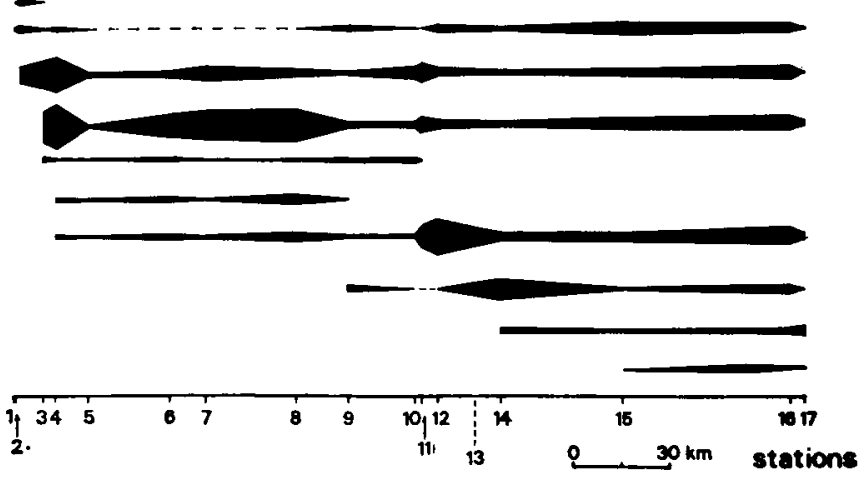

FiG. 2. - Variation longitudinale de l'abondance relative des Mollusques de l'Aube (5 espèces présentant des abondances notables seulement au niveau de la noue de Magnicourt, station 13, ne figurent pas sur le graphique). 
cours de l'analyse d'autres groupes faunistiques: Plécoptères, Ephéméroptères, Trichoptères, Poissons (Verneaux, 1973), ou de Diatomées benthiques (Coste, 1977).

Cinq groupements principaux s'individualisent le long de cette structure, matérialisée par la jonction des stations classées en fonction de leur peuplement malacologique dans l'ordre de leur succession le long du cours d'eau; les espèces présentant les plus fortes contributions aux axes considérées comme espèces « repères " sont soulignées sur le graphique (fig 3).

$B_{8}$

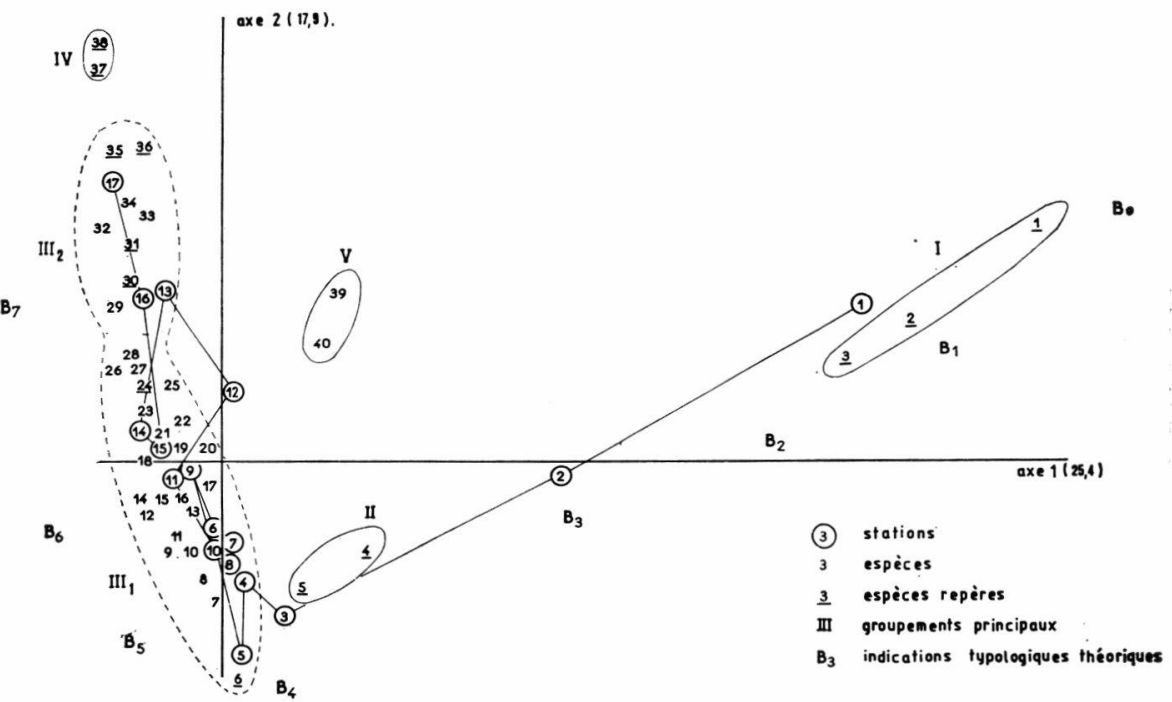

Fig. 3. - Analyse factorielle des correspondances espèces-stations : structure biologique de l'Aube en fonction de la répartition des Mollusques $(43,4 \%$ d'inertie expliquée pour les deux premiers axes $\left.\mathrm{F}_{1} \mathrm{~F}_{2}\right)$.

Nous analyserons en premier lieu les trois groupements qui s'individualisent nettement de la distribution :

- GRoupement DES Sources (groupement I) (station 1)

Bythinella carinulata, Pisidium personatum, Bythiospeum (Lartetia) diaphanum. Nettement séparé du reste de la distribution, ce groupement renferme une espèce appartenant au domaine des eaux souterraines ( $B$. diaphanum) présente épisodiquement sur la station, notamment après les crues. Bien que la sténothermie de $P$. personatum soit contestée par certains auteurs (Favre 1929, Kuhn 1940, Feldmann 1977), elle semble admise par d'autres (Kuiper com. pers.).

- GROUPEMENT IV (station 17)

Potamopyrgus jenkinsi, Viviparus viviparus. Ces deux dernières 
espèces n'ont été récoltées qu'à la sortie de l'ancien canal de Haute. Seine, à l'amont de la confluence Aube-Seine. Ce plan d'eau, non soumis au régime d'écoulement des caux de la rivière, est assimilable à un systìme d'eau calme présentant une température estivale plus élevée (température août 1977 : Aube $18,5^{\circ} \mathrm{C}$, sortie canal $22^{\circ} \mathrm{C}$ ).

\section{- GROUPEMENT V (station 12)}

Pisidium casertanum, Galba truncatula. Ces deux dernières espèces qui présentent une abondance notable, uniquement aux deux extrémités du cours d'eau constituent un groupement particulier bien isolé sur le graphique. Les auteurs s'accordent pour les qualifier d'eurytopes (Boycott 1936, Dittmar 1955, Kuiper 1964), bien que certains aient souligné le caractère crénophile de G. truncatula (Thienemann 1926, Kuhn 1940, Nadig 1942, Illies 1952).

En fait, cette espèce peut se développer en abondance dans tous les milieux aquatiques, fussent-ils temporaires. Dans les rivières et les fleuves, son habitat est limité aux bordures, parfois abondamment colonisées, fréquemment hors de l'eau (espèce amphibie).

$\square$ Le continuum principal, allongé, constitué par la majorité des taxons, s'organise comme une succession continue des espèces ou des noyaux d'espèces formant un continuum a noda exprimant leur remplacement progressif les unes par les autres le long du cours d'eau ; toutefois, deux groupements principaux peuvent être reconnus:

- GROUPEMENT DU COURS SUPÉRIEUR (groupement II) (stations 2 et 3)

Radix peregra, Ancylus fluviatilis. Ce sont les seuls représentants des secteurs à écoulement rapide et à forte granulométrie ; ces espèces, conformément aux remarques effectuées par Illies (1955), sont d'ailleurs peu représentées sur l'Aube, qui prend sa source à faible altitude.

- GROUPEMENT III (constitué par deux sous-groupements)

1) sous-groupement du cours moyen (stations 4 à 15 sauf 13)

Ce secteur renferme essentiellement des espèces à faibles contributions aux axes, largement distribuées sur l'Aube, notamment les espèces des troisième et quatrième sous-groupements.

On distingue quatre noyaux :

a) Bathiomphalus contortus (station 5).

b) Pisidium amnicum, Valvata piscinalis (5 espèces, stations 4-6-7-810). Selon Thienemann (1911-1912) et Fisher (1920) P. amnicum serait caractéristique de la "zone à Ombre".

c) Pisidium nitidum, Physa fontinalis (11 espèces, stations 9-11-1415). 
d) Unio crassus, Acroloxus lacustris, Bithynia tentaculata (6 espèces, station 14).

2) sous-groupement $d u$ cours inférieur (stations 13-16-17) dans lequel on distingue 3 noyaux :

a) Unio pictorum, Radix auricularia.

b) Pisidium supinum (4 espèces).

c) Pisidium moitessierianum, Armiger crista.

A la suite de nombreux auteurs (Thienemann 1950, Bogoescu 1952, Ross 1956, Botosaneanu 1960, Verneaux 1973) il convient de constater que les espèces hautement singificatives appartiennent aux groupements extrêmes alors que les groupements plus centraux, à caractère eurytope, en sont pratiquement dépourvus. Le degré d'euryecie des espèces, les possibilités typologiques des Mollusques aquatiques peuvent ainsi être appréhendés.

Les proximités entre espèces et stations qui apparaissent immédiatement sur le graphique à deux dimensions font ressortir les affinités existant entre elles.

La succession des espèces ou groupements d'espèces permet d'apprécier l'ampleur du phénomène de vicariance au sein de l'embranchement des Mollusques, tant en ce qui concerne les gastéropodes, que les bivalves. Il est, par ailleurs, intéressant de noter que ce sont les Pisidies à fortes charnières ( $P$. ponderosum, et appendiculés, $P$. supinum, $P$. moitessierianum, $P$. henslowanum), qui dominent dans le tronçon inférieur de l'Aube.

\section{REMARQUES AU NIVEAU DU COURS D'EAU}

Comme l'a montré Verneaux (1973), chaque écosystème d'eau courante possède une structure biologique propre exprimant à la fois les particularités du milieu, en particulier les accidents écologiques, et celles de son peuplement. Les modifications fréquentes de la courbe structurale de l'Aube par rapport à une structure typique théorique continue et progressive (fig. 3) résultent d'accidents écologiques naturels : rupture de pente (station 4), noue (station 14), ou artificiels : retenue et déversoir pour l'alimentation d'un moulin (station 8), retenue de Beaulieu (station 10), pollution organique (station 15, aval de la confluence Aube - Voire), simplification de l'habitat (station 12). Le peuplement de cette dernière station qui s'éloigne notablement de la courbe théorique, est dominé par des espèces eurytopes présentant des abondances très faibles.

\section{DISCUSSION ET CONCLUSIONS}

Comme l'indiquent les données typologiques obtenues à l'aide de composantes physiques relevées sur chaque station prospectée, l'Aube, 
hormis la zone des sources et le secteur terminal, s'apparente au Rhithron. La faible représentation du Potamon, plus favorable au déve. loppement des Mollusques (diversité et biomasse) explique l'absence ou la faible abondance de certaines espèces. Par ailleurs, ce fait retentit sur la position des espèces potamiques dans le plan de l'analyse factorielle : ainsi Physa acuta, Anodonta anatina, Unio pictorum devraient très probablement occuper des positions plus périphériques dans le cas d'une analyse typologique traitant d'un ensemble de systèmcs clifférents.

D'après les données recueillies en d'autres lieux (bassin du Rhône, Dordogne) il est probable qu'un ensemble d'informations plus diversifiées et plus complètes se traduirait par une dissociation de la plupart des espèces du quatrième noyau du sous-groupement III-1 quj ne sont euryèces qu'à l'échelle de l'Aube. En fait, le seul groupement répertorié susceptible d'être réellement euryèce dans un écosystème d'eau courante complet serait constitué par $P$. casertanum et $G$. truncatula (groupement $\mathrm{V}$, nettement détaché du continuum).

A titre indicatif, l'application de la formule de repérage de l'appartenance typologique théorique d'une station (Verneaux 1977) permet de situer les groupements dans un cadre théorique (Tableau IV).

\begin{tabular}{|c|c|c|c|}
\hline $\begin{array}{l}\text { Groupements d'espèces } \\
\text { de mollusques }\end{array}$ & \multicolumn{2}{|c|}{$\begin{array}{l}\text { Niveaux typologiques } \\
\text { calculés selon } \\
\text { Verneaux (1977) }\end{array}$} & $\begin{array}{c}\begin{array}{c}\text { Espèces repères associées } \\
\text { dans le cas de l'Aube }\end{array} \\
\text { B. diaphanum }\end{array}$ \\
\hline Groupement des sources (I) & Crenon & $\begin{array}{l}\mathrm{B}_{0} \\
\mathrm{~B}_{1}\end{array}$ & $\begin{array}{l}\text { B. diaphanum } \\
\text { B. carinulata } \\
\text { P. personatum }\end{array}$ \\
\hline $\begin{array}{l}\text { Groupement du cours supé- } \\
\text { rieur (II) }\end{array}$ & & $\begin{array}{l}\mathbf{B}_{\vec{S}} \\
\mathbf{B}_{3}\end{array}$ & $\begin{array}{l}\text { R. peregra } \\
\text { A. fluviatilis }\end{array}$ \\
\hline $\begin{array}{l}\text { Groupement du cours moyen } \\
\text { (sous-groupement III-1-a-b) }\end{array}$ & RHITHRON & $\begin{array}{l}\mathrm{B}_{4} \\
\mathrm{~B}_{3}\end{array}$ & $\begin{array}{l}\text { B. contortus } \\
P . \text { amnicum } \\
V . \text { piscinalis }\end{array}$ \\
\hline \multirow[t]{2}{*}{$\begin{array}{l}\text { Groupement du cours infé- } \\
\text { rieur } \\
\text { (sous-groupcment III-1-d- } \\
\text { III-2, groupement IV) }\end{array}$} & Potamon & $\begin{array}{l}B_{6} \\
B_{7}\end{array}$ & $\begin{array}{l}U . \text { crassus } \\
R . \text { auricularia } \\
\text { A. crista } \\
P . \text { supinum } \\
P . \text { moitessierianum }\end{array}$ \\
\hline & & $\mathbf{B}_{\mathbf{z}}$ & $\begin{array}{l}V . \text { viviparus } \\
P . \text { jenkinsi }\end{array}$ \\
\hline
\end{tabular}

Tableau IV. - Peuplement malacologique des niveaux typologiques de l'Aube.

Aux différents niveaux typologiques successifs correspondent des espèces ou des groupements d'espèces à forte contribution aux axes, 
considérés comme les repères de l'organisation malacologique de l'Aube (ce qui exclut les espèces centrales). En se rapportant à la distribution des espèces sur les stations (graphique 2) on peut distinguer parmi les espèces caractéristiques :

- des espèces sténoèces, typologiquement les plus significatives, liées à un biotope précis comme les sources: B. diaphanum, B. carinulata.

- des espèces ayant une distribution longitudinale de plus grande amplitude dont les preferendums se succèdent statistiquement: espèces des niveaux $\mathrm{B}_{4}$ à $\mathrm{B}_{8}: B$. contortus - $P$. amnicum $-V$. piscinalis - $P$. nitidum - $P$. fontinalis - $U$. crassus - A. lacustris - B. tentaculata - U. pictorum - $R$. auricularia - P. supinum - P. moitessierianum - A. crista. Cette succession représente l'essentiel de la structure malacologique de l'Aube.

- des espèces à distribution plus large encore, mais dont la position dans l'analyse est définie par l'absence d'autres espèces; c'est le cas de $A$. fluviatilis et $R$. peregra qui colonisent le secteur compris entre l'aval des sources et l'amont d'Auberive (ferme «la Thulière ») à l'exclusion des autres espèces.

L'existence de discontinuités statistiques dans la distribution des espèces a permis d'isoler plusieurs groupements; toutefois, l'intérêt d'une telle analyse n'est pas de faire apparaître des noyaux (un système théorique parfait n'en présenterait d'ailleurs pas) mais plutôt de proposer une hiérarchisation des espèces le long de l'écosystème permettant de préciser pour chacune d'elle :

- son preferendum typologique (appartenance de l'espèce à un niveau donné de la succession des espèces),

- son amplitude typologique (associée à son degré d'euryécie, proportionnelle au nombre de types écologiques dans lesquels l'espèce est significativement présente.

Cette approche ne peut être effectuée qu'à partir de l'analyse des peuplements de systèmes aquatiques nombreux et très différenciés écologiquement, elle constitue l'objet de nos travaux actuels.

\section{TRAVAUX CITÉS}

Albrecht (M. L.). 1953. - Die Plane und andere Flämingbäche (Ein Beitrag zur Kenntnis der Fliessgewässer der Endmoränenzüge des Norddeutschen Tiefebene. Z. Fisch. Hilfswiss (N. F.), 1, 390-473.

Armitage (P. D.), Mc Hale (A.M.) et CRisp (D. C.). 1975. - A survey of the invertebrates of four streams in the Moor House National Nature Reserve in Northern England. Freshw. Biol., 5, 479-495.

Benzecri (J. P.). 1973. - L'analyse des données. Dunod éd., Paris, 2 vol., 1248 p. 
Bernasconi (R.). 1967. - Les Hydrobiinae cavernicoles de Suisse et des régions limitrophes. I. Aperçu historique et distribution zoogéographique. Ann. Spéléo., 3, 522-535.

Bernasconi (R.). 1969. - Les Hydrobiinae cavernicoles de Suisse et des régions limitrophes. II. Distribution zoogéographique et limite septentrionale de la zone à Bythiospeum. Ann. Spéléo., 24 (4) : 683-694.

Bernasconi (R.). 1972. - Les Hydrobiidae cavernicoles de Suisse et des régions limitrophes. III. Essai de révision des Bythiospeum. Ann. Spéléo., 27 (4) : 761-772.

Bernasconi (R.). 1975. - Les Hydrobiidae cavernicoles de Suisse et des régions limitrophes. V. Révision des Hauffenia Pollonera. Ann. Spéléo., 30 (2) : $303-$ 311.

Beyer (H.). 1932. - Die Tierwelt der Bäche und Quellen des Baumbergegebietes. Abh. Westf. Prov. Mus. Naturkunde, 3 : 1-185.

Bishop (M. J.) et Hewirt (S. J.). 1976. - Assemblages of Pisidium species from localitics in eastern England. Fresh. Biol., 6: 177-182.

BlesSCH (J. Von). 1977. - Bodenfaunistiche Untersuchungen in Aare und Rhein. Schweiz. Z. Hydrol., 39 (1) : 46-68.

Bceters (H. D.). 1973. - Die Gattung Bythinella und die Gattung Marstoniopsis in Westeuropa 1 - Westeuropäische Hydrobiidae. Malacologia, 14 : 271-285.

Bogoescu (C.). 1952. - L'influence de la température et de la vitesse de l'eau sur la répartition des espèces des Ephéméroptères. Rev. Univ. C. I. Parhon. Stiint. Natur., $1: 9$ p.

Bonazzi (G.) et GheTri (P. F.). 1977. - I macroinvertebrati del torrente Parma risultati di un ciclo annuale di recerche Atenco Parmense. Acta Nat., 13 (3) : 351-395.

Bornhauser (K.). 1912. - Die Tierwelt der Quellen und Bäche in der Umgebung Basels. Internat. Rev. Ges. Hydrobiol. Biol. Suppl., 5 : 1-90.

Botosaneanu (L.) et Negrea (A.). 1976. - Sur quelques aspects biologiques d'une rivière des Monts du Banat - Roumanie: La Nora (et surtout sur une remarquable association de Gastéropodes). Rivista di idrobiologia, XV, 3 : 403-432.

Boycort (A. E.). 1936. - The habitats of freshwater Mollusca in Britain. J. Anim. Ecol., 5 : 116-186.

CALow (P.). 1973. - Field observations and laboratory experiments on the general food requirements of two species of freshwater snail, Planorbis contortus Linn. and Ancylus fluviatilis Mull. Proc. Malac. Soc. Lond., 40 : 483-489.

CaLow (P.). 1974. - Evidence for bacterial feeding in Planorbis contortus Linn. Proc. Malac. Soc. Lond., 41 : 145-156.

CALOW (P.). 1975. - Cellulase activity and niche separation in freswhater Gastropods. Nature, 255, (5 508) : 478-480.

Cherbonnier (G.). 1952. - Note sur Pseudamnicola lanceolata. Bull. Muséum d'Hist. Nat., $24: 568-569$.

CoRdier (B.). 1965. - L'analyse factorielle des correspondances. Thèse $3^{e}$ Cycle, Univ. Rennes, $66 \mathrm{p}$.

Coste (M.). 1978. - Sur l'utilisation des Diatomées benthiques pour l'appréciation de la qualité biologique des eaux courantes. Thèse $3^{\circ}$ Cycle, Univ. Besançon, $143 \mathrm{p}$.

Craw Mc (M. B.). 1970. - Aspects of the growth of the snail Lymnaea palustris. Malacologia. 10 (2) : 399-413.

Dance (S. P). 1957. - Notes on the Pisidium fauna of the Pevensey levels district, with special reference to Pisidium pseudosphaerium Favre. J. Conch., 27: 177-181.

Dance (S. P.). 1970. - Pisidium lilljeborgii in the river Teif, West Wales. J. Conch., $27: 177-181$. 
Ditrmar (H.). 1955. - Ein Sauerlandbach Untersuchungen an einem Wiesen - Mit. tel gebirgsbach. Arch. Hydrobiol., $50: 307-544$.

Durrant (M. P.). 1977. - Some factors that affect the distribution of Ancylus in the river systems of Great Britain. J. Moll. Stud., 43 : 67-78.

FAVRE (J.). 1927. - Les Mollusques post-glaciaires et actuels du Bassin de Genève. Mémoire de la Société de Physique et d'Histoire Naturelle de Genève, 40 (3) : 169-434.

FeldmanN (R.). 1971. - Die Kleinmuscheln (Spaeriidae) des mittleren Ruhrtales. Decheniana, $123(1 / 2)$ : 27-47.

FeldmanN (R.). 1972. - Die Süsswassermollusken des Messtischblattes Menden (Sauerland). Dortmunder Beträge zur Landeskunde, Naturw. Mitteilungen, $6: 45-56$.

Feldmann (R.). 1975. - Die Molluskenfauna der Quellsümpfe (Helokrenen) im sudwestfälischen Bergland. Decheniana, 127 : 135-143.

FeldmanN (R.). 1977. - Die Kleinmuschelfauna des Südwestfälischen Berglandes. Ein Beitrag zur Faunistik und ökologie der Sphaeriidae. Abhandlungen aus dem Landesmuseum für Naturkunde Münster. 39 (1/) : 40-57.

Fellenberg (W. O.). 1968. - Zur Süsswassermolluskenfauna des Sauerlandes. Abhandlungen aus dem landesmuseum für Naturkunde Münster, $30: 3-22$.

FitrkaU (J.). 1949. - Mitteilung über die in der Fulda und ihren Zuflüssen aufgefundenen Weichtiere. Jahresber. d. Limnologischen Station Freudenthal. $S: 17-19$.

Fisher (A.). 1920. - Die "Aschenregion der Diemel ". Inaug. Diss. Münster. 63 p.

Frömming (E.). 1950. - Physa acuta Drap. in Berlin eingebürgert. Archiv. für Hydrobiol., 10 (3) : 304-316.

GeYER (D.). 1911. - Die Molluskenfauna des Neckars. Jahreshefte des Vereins für Vaterländische Naturkunde in Württemberg: 354-371.

GEIJSKES (D. C.). 1936. - Faunistich-ökologische Untersuchungen am Röserenbach bei Liestal im Baseler Tafeljura. T. Entomol., 78 : 251-382.

Haukioja (E.) et HaKala (T.). 1974. - Vertical distribution of freshwater mussels (Unionidae) in South-Western Finland. Ann. Zool. Fennici, 11: 127-130.

Hairston (N.) et HubEN Dick (B.). 1958. - An evaluation of techniques used in estimating snail populations. Bull. Org. Mond. Santé, 19:661-672.

HässLein (L.). 1956. - Mollusken und Molluskengesellschaften der Gewässer dcs Nördlinger Rieses. Jh. Ver. vaterl. Naturk. Wurttemberg, 111 (1) : 174-199.

Hendelberg (J.). 1959. - The freshwater pearl mussel, Margaritifera margaritifera. Institute of freshwater Research. Drottningholm Report, $4: 149-170$.

Hinz (W.). 1968. - Die Süsswasser Molluskenfauna von Castrop-Rauxel. Gewässer und Abwässer, $46: 13-19$.

Illies (J.). 1952. - Die Mölle - Faunistich ökologische Untersuchungen an einem Forellenbach im Lipper Bergland. Arch für Hydrobiol., 46 : 424-612.

ILlies (J.). 1955. - Der biologische Aspekt der limnologischen Fliesswassertypisierung. Arch. für Hydrobiol. Suppl. 22 : 337-346.

JuncBlutil (J. H.). 1972. - Die Verbreitung und ökologie des Rassenkreises Bythinella dunkeri. Arch. für Hydrobiol., 70 (2) : 230-273.

JunGbluti (J. H.) et LehmanN (G.). 1976. - Untersuchungen zur Verbreitung, Morphologie und okologie der Margaritifera Populationem an den atypischen Standorten des jungtertiären Basaltes im Vogelsberg. Arch. für Hydrobiol., 78 (2) : 165-212.

Kinzelbach (R.). 1972. - Einschleppung und Einwanderung von Wirbellosen in Ober und MittleRhein. Mz. Naturev. Arch., 11, S : 109-150.

KinZelbach (R.) und coll. 1972. - Bestandsrückgang der Schneckenfauna des Rheins zwischen Strassburg und Koblenz. Natur und Museum, 102 (6) : 197206.

KUHN (G.). 1940. - Zur ökologie und Biologie der Gewässer (Quellen und Abflüsse) des Wassersprengs bei Wien. Arch. für Hydrobiol., 36 : 157-262. 
KuIPER. (J. G. J.). 1949. - Pisidium pseudosphaerium Favre in England. J. Conch., 23 (2) : 27-32.

Kuiper (J. G. J.). 1964. - Sur la présence des espèces du genre Pisidium dans les eaux lacustres des Pyrénées-Orientales. Vie et Milieu, 15 (3) : 677-685.

KUIPER (J. G. J.) et WoLFF (W. J.). 1970. - The Mollusca of the estuarine region of the rivers Rhine, Meuse and Scheldt in relation to the hydrography of the area. III. The genus Pisidium. Basteria, 34 (1-2) : 1-42.

LAdLe (L.) et BARON (F.). 1969. - Studies on three species of Pisidium (Mollusca bivalva) from a chalk stream. J. Anim. Ecol., 38: 407-413.

LeBART (L.) et FÉNELON (J. P.). 1971. - Statistique et informatique appliquées. Dunod éd., Paris : $426 \mathrm{p}$.

Lucas (A.). 1960. - Remarques sur l'écologie d'Hydrobia jenkinsi en France. $J$. Conch. C: 121-128.

Maitland (P. S.). 1965. - Notes on the biology of Ancylus fluviatilis in the river Endrick, Scotland. Proc. Malac. Soc. Land., $36:$ 339-347.

MEIER-BRook (C.). 1975. - Der ökologische Indikatorwert mitteleuropäischer Pisidium. Arten (Mollusca, Eulamellibranchiata). Eiszeitalter u. Gegenwart, 26 : 190-195.

Miegel (H. Von). 1961. - Die Molluskenfauna des Xantener Altrheins. Gewässer und Abwässer, $29:$ 7-12.

Miegel (H. Von). 1964. - Süsswasser-Mollusken des Rheingebietes. Gewässer und Abwässer, $33: 1-75$.

Mentzen (R.). 1926. - Bemerkungen zur Biologie und Ökologie der Mitteleuropäischen Unionidae. Arch. für Hydrobiol., 17 : 380-394.

Modell (M.). 1974. - Die Najaden des Neckar-Gebietes (Bivalvia, Unionacea). Veröff. Zoll. Staatssamml. München, B 17, S : 109-138.

NaDIG (A.). 1942. - Hydrobiologische Untersuchungen in Quellen des schweizerischen Nationalparkes im Engadin. Ergebn. d. Wissensch. Unters. d. Schweiz. Nationalpark, 1, S : 267-432.

Negus (C. L.). 1966. - A quantitative study of growth and production of Unionid Mussels in the river Thames ad Reading. J. anim. Ecol., $35: 513-532$.

NiETZKE (G.). 1937. - Die Kossau hydrobiologisch-faunistische Untersuchungen an schleswig-holsteinischen Fliessgewässern. Arch. für Hydrobiol., 32 : 1-74.

Norelius (I.). 1967. - Age groups and habitat of Unionid mussels in a South Swedish stream. Oikos, $18: 365-368$.

Puton (E.). 1847. - Les Mollusques terrestres et fluviatiles des Vosges. Epinal, $104 \mathrm{p}$

RADoman (P.). 1976. - Speciation within the family Bythinellidae on the Balkans and Asia Minor. Z. Zool. Systemat. Evol. Forsch., 14 (2) : 130-152.

Ross (H.). 1956. - Evolution and classification of the moutain caddiflies. Univ. Ill. Press. Ed. Urbana : 213 p.

SCHÄFER (H. Von). 1953. - Untersuchungen zur ökologie von Bithynia tentaculata. Arch. Moll., 82 (1/3) : 67-70.

SCHERmer (E.). 1922. - Die Mollusken einiger norddeutscher Quellgebiete. Arch. für MolluskenKunde, $6: 166-180$.

Schuster (O.). 1925. - Postglaziale Quellkalke Schleswig-Holstein und ihre Mol luskenfauna in Beziehung zu den Veränderungen des Klimas und der Gewässer. Arch. für Hydrobiol., $16: 1-73$.

SHADIN (V.J.). 1935. - Uber die ökologische und geographische Verbreitung der Süsswassermollusken in der U.d.S.S.R. Zoogeographica, $2: 295-554$.

Thiel (K. D.) et SubkLew (H. J.). 1976. - Der Bierbach in Naturschutzgebiet \& El. dena » bei Greifswald. Limnologica, 11 (1) : 101-123.

ThienemanN (A.). 1911-1912. - Beiträge zur Kenntnis der Westf. Süsswasserfauna IV. Die Tierwelt der Bäche des Sauerlandes. $40 \mathrm{ahr}$. West. Prov. Verf. f. Wiss. u. Kunst., S : 43-83. 
Thienemann (A.). 1926. - Hydrobiologische Untersuchungen an der kalten Quellen and Bächen der Halbinsel Jasmud auf Rügen. Arch. für Hydrobiol., 17, S : 221-336.

Thienemann (A.). 1950. - Verbreitungsgeschichte der Süsswassertierwelt Europas. Die Binnengewässer, $18: 809 \mathrm{p}$.

Tomaszewski (W.). 1928. - Beitrag zur Kenntnis der Tierwelt schlesischer Bergbäche. Abh. d. Naturf. Ges. Görlitz., 31 H. 2, S : 1-86.

Verneaux (J.). 1973. - Cours d'eau de Franche-Comté (Massif du Jura). Recherches écologiques sur le réseau hydrographique du Doubs. Essai de biotypologie. Thèse Doct. ès Sci. Nat. Fac. Sci. Univ. Besançon, 257 p.

Verneaux (J.). 1976. - Biotypologie de l'écosystème "eau courante ". La structure biotypologique. C. R. Acad. Sc. Paris, t. 283 D, 1663-1666.

Verneaux (J.). 1977. - Biotypologie de l'écosystème «eau courante ». Déterminisme approché de la structure biotypologique. C. R. Acad. Sc. Paris, t. $284 \mathrm{D}, 77-79$.

WoLfF (W. J.). 1968. - The Mollusca of the estuarine region of the rivers Rhine, Meuse and Scheldt in relation to the hydrography of the area. I. Les Unionidae. Basteria, $32: 13-47$.

Young (M.). 1975. - The life cycles of six species of freshwater molluscs in the Worcester - Birmingham Canal. Proc. Malac. Soc. Land., 41 : 533-548.

Rapport C.T.G.R.E.F. 1978. - Etude hydrobiologique de l'Aube. Diagnose préalable à la réalisation d'un barrage réservoir, $\mathrm{n}^{\circ} 35,71 \mathrm{p}$.

Février 1980. 